\title{
Electrophoresis of a Sphere Normal to a Plane at Arbitrary Electrical Potential and Double Layer Thickness
}

\author{
Ming-Hui Chih, Eric Lee, and Jyh-Ping Hsu ${ }^{1}$ \\ Department of Chemical Engineering, National Taiwan University, Taipei, Taiwan 10617, Republic of China
}

Received June 15, 2001; accepted January 18, 2002; published online March 13, 2002

\begin{abstract}
The electrophoretic movement of a sphere normal to an uncharged, planar surface is analyzed theoretically, taking the effect of double layer polarization into account. Here, both the surface potential of the particle and the thickness of the double layer surrounding it can be arbitrary. We show that if double layer polarization is neglected, the effect of the surface potential of a particle on its electrophoretic velocity is inappreciable. On the contrary, it becomes significant if double layer polarization is present. However, if the distance between the particle and the surface is sufficiently close, since the hydrodynamic effect dominates, the influence of the surface potential and double layer polarization becomes insignificant. (c) 2002 Elsevier Science (USA)
\end{abstract}

\section{INTRODUCTION}

The presence of a boundary on the electrophoretic behavior of a charged entity is of fundamental significance and draws the attention of many researches in the past two decades. Typical examples include the electrophoresis of a particle through a pore or in a porous medium and that of a concentrated dispersion. Zydeny (1) and Lee et al. (2, 3), for instance, considered the electrophoretic behavior of a sphere in a spherical cavity. Morrison and Stukel (4) investigated the electrophoresis of a sphere normal to a plane under the conditions of thin double layer and low surface potential. Bispherical coordinates were chosen for the description of the electrokinetic equations. They found that the plane has the effect of retarding the movement of the particle, and the closer the particle to the plane the more significant it is. Pointing out that the analysis of Morrison and Stukel is incorrect, Keh and Lien (5) reanalyzed the problem. Keh and Anderson (6) considered the electrophoretic behavior of a sphere for the cases in which it moves normal to a plane, parallel to a plane, and along the axis of a cylindrical cavity. Analytical solution was derived based upon a reflection method under the conditions of low electrical potential and thin double layer. It was found that an electroosmotic effect will be induced by a charged boundary. The same problem was discussed by

\footnotetext{
${ }^{1}$ To whom correspondence should be addressed. E-mail: jphsu@ ccms.ntu. edu.tw.
}

Ennis and Anderson (7) through considering a double layer of finite thickness, but it cannot reach the boundary. The problem that an arbitrary prolate body of revolution moves normal to a conducting plane for the case of thin double layer was analyzed by Feng and $\mathrm{Wu}(8)$. The electrophoresis of a particle normal to a plane at low electrical potential but arbitrary double layer thickness was considered by Shugai and Carnie (9). Recently, Tang et al. (10) analyzed the electrophoretic behavior of a charge-regulated sphere normal to a plane at low electrical potential.

The electrophoresis of spherical particles has been studied extensively (see, e.g., (11-24)). Most of the results reported, however, are limited to special cases such as low electrical potential and thin double layer. This is mainly due to the nonlinear nature of the governing electrokinetic equations, and solving them analytically becomes almost impossible. For the case of a sphere which moves normal to a planar surface, previous analyses are restricted to low surface potential and the effect of double layer polarization (DLP) is negligible. In this work, these limitations are removed, and the general electrokinetic equations are solved numerically.

\section{THEORY}

Referring to Fig. 1, we consider the electrophoresis of a sphere of radius $a$ normal to an uncharged plane subject to a uniform electric field $\mathbf{E}_{z}$ with strength $E_{z}$ in the $Z$ direction. Let $h$ be the distance between the center of the particle and the surface. The liquid phase contains $z_{1}: z_{2}$ electrolyte solution, $z_{1}$ and $z_{2}$ being respectively the valences of cations and anions, with $n_{20}=n_{10} / \alpha, n_{10}$ and $n_{20}$ being respectively the bulk concentrations of cations and anions. Suppose that the velocity of the particle $\mathbf{U}$ is slow so that a quasi-steady state can be assumed. The bispherical coordinates $(\xi, \eta, \phi)$ are adopted with $\eta=0$ and $\eta=\eta_{0}$ represent respectively the planar surface and the surface of the particle. The symmetric nature of the problem suggests that only half of the $(\xi, \eta)$ domain needs to be considered. The bispherical coordinates used are related to the cylindrical coordinates $(r, \theta, Z)$ by $(25)$

$$
Z=c \frac{\sinh \eta}{\cosh \eta-\cos \xi}
$$




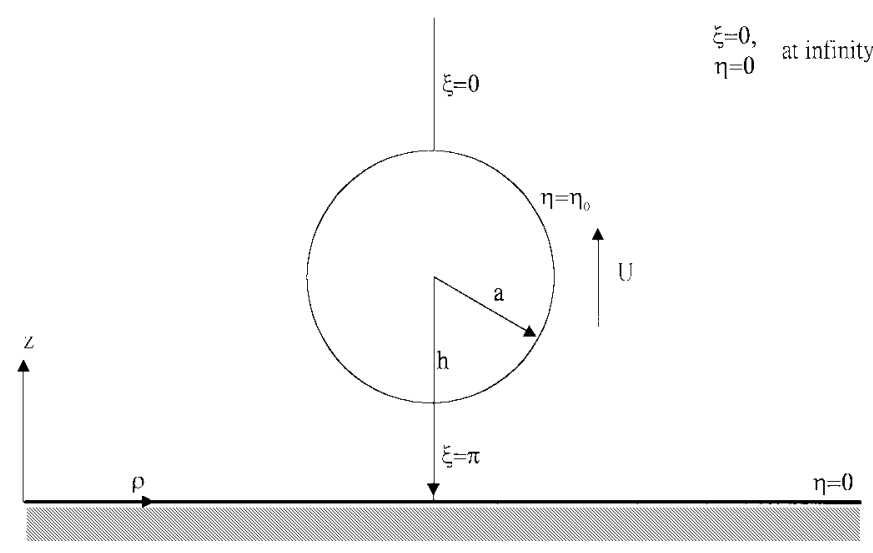

FIG. 1. The bispherical coordinates $(\xi, \eta, \phi)$ adopted where $\eta=0$ and $\eta=$ $\eta_{0}$ represent respectively the plane and the surface of particle, $a$ is particle radius, and $h$ is the distance between the center of the particle and the plane. A uniform electric field $\mathbf{E}_{z}$ is applied in the $Z$ direction, and $\mathbf{U}$ is the velocity of particle.

$$
\rho=c \frac{\sin \xi}{\cosh \eta-\cos \xi},
$$

where $c$ is the focal length and $0 \leq \eta \leq \eta_{0}, 0 \leq \xi \leq \pi, \eta_{0}=$ $\cosh ^{-1}(h / a)$.

The elctrokinetic equations of the present problem comprise the governing equations for ion conservation, electrical field, and flow field. The conservation of ions in the liquid phase yields

$$
\nabla \cdot\left[D_{j}\left[\nabla n_{j}+\frac{z_{j} e n_{j}}{k_{B} T} \nabla \phi\right]-n_{j} v\right]=0 \quad j=1,2,
$$

where $\nabla$ is the gradient operator, $n_{j}, D_{j}$, and $z_{j}$ are respectively the number concentration, the diffusivity, and the valence of ion species $j, e$, and $\phi$ are the elementary charge and the electrical potential respectively, $\mathbf{v}$ is the fluid velocity, and $k_{B}$ and $T$ are respectively the Boltzmann constant and the absolute temperature. Suppose that the electrical field can be described by the Poission equation

$$
\nabla^{2} \phi=-\frac{\rho_{e}}{\varepsilon}=-\sum_{j=1}^{2} \frac{z_{j} e n_{j}}{\varepsilon},
$$

where $\rho_{e}$ is the space charge density, and $\varepsilon$ is the permittivity of the liquid phase. We consider incompressible fluid with constant physical properties. Assume that the flow field can be described by the Navier-Stokes equation

$$
\begin{gathered}
\nabla \cdot \mathbf{v}=0 \\
\mu \nabla^{2} \mathbf{v}-\nabla p-\rho_{e} \nabla \phi=0 .
\end{gathered}
$$

In these expressions $p$ is the pressure and $\mu$ is the viscosity.

For convenience, $\phi$ is decomposed into the sum of the electrical potential in the absence of the applied electric field (or the equilibrium electrical potential) $\phi_{1}$, and a perturbed electrical potential $\phi_{2}$, which arises from the applied electric field (16). The distortion of the double layer surrounding the particle can be simulated by

$$
n_{j}=n_{j 0} \exp \left(-\frac{z_{j} e\left(\phi_{1}+\phi_{2}+g_{j}\right)}{k_{B} T}\right), \quad j=1,2,
$$

where $g_{j}$ is a perturbed function. Suppose that the applied electric field is weak, and $\phi_{2}$ and $g_{j}$ are much smaller than $\phi_{1}$. In this case Eq. [7] can be approximated by

$n_{j}=n_{j 0} \exp \left(-\frac{z_{j} e}{k_{B} T} \phi_{1}\right)\left[1-\frac{z_{j} e}{k_{B} T}\left(\phi_{2}+g_{j}\right)\right], \quad j=1,2$

and the space charge density can be approximated by

$$
\rho \cong \sum_{j=1}^{2} z_{j} e n_{j 0} \exp \left(-\frac{z_{j} e}{k_{B} T} \phi_{1}\right)\left[1-\frac{z_{j} e}{k_{B} T}\left(\phi_{2}+g_{j}\right)\right] \text {. }
$$

For a simpler mathematical manipulation, we define the scaled quantities $\phi_{j}^{*}=\phi_{j} / \zeta_{a}, g_{j}^{*}=g_{j} / \zeta_{a}$, and $n_{j}^{*}=n_{j} / n_{10}$, where $\zeta_{a}$ is the electrical potential on particle surface. In scaled quantities the equilibrium electrical potential can be expressed as

$$
\nabla^{* 2} \phi_{1}^{*}=-\frac{1}{(1+\alpha)} \frac{(\kappa a)^{2}}{\phi_{r}}\left[\exp \left(-\phi_{r} \phi_{1}^{*}\right)-\exp \left(\alpha \phi_{r} \phi_{1}^{*}\right)\right]
$$

where $\phi_{r}=\zeta_{a} /\left(z_{1} e / k_{B} T\right)$ is the scaled surface potential of the particle, and the reciprocal Debye length $\kappa$ and the scaled gradient operator $\nabla^{* 2}$ are defined by

$$
\begin{aligned}
\kappa & =\left[\sum_{j=1}^{2} n_{j 0}\left(e z_{j}\right)^{2} / \varepsilon k_{B} T\right]^{1 / 2} \\
\nabla^{* 2} & =\frac{x^{2}}{c^{* 2}}\left[\frac{\partial^{2}}{\partial \eta^{2}}+\frac{\partial^{2}}{\partial \xi^{2}}-\frac{\sinh \eta}{x} \frac{\partial}{\partial \eta}+\frac{\cos \xi \cosh \eta-1}{x \sin \xi} \frac{\partial}{\partial \xi}\right] .
\end{aligned}
$$

In these expression $c^{*}=c / a$, and $x=\cosh \eta-\cos \xi$.

Suppose that the equilibrium electrical potential vanishes at a point far away from particle surface. Then

$$
\phi_{1}^{*}=0, \quad \eta=0 \quad \text { and } \quad \xi=0, \text { or } \quad \eta=0 .
$$

The symmetric nature of the problem implies that

$$
\frac{\partial \phi_{1}^{*}}{\partial \xi}=0, \quad \xi=0 \quad \text { or } \quad \xi=\pi
$$

Assuming constant electrical potential on particle surface, we have

$$
\phi_{1}^{*}=1, \quad \eta=\eta_{0}
$$


Employing the relation $\phi=\phi_{1}+\phi_{2}$, Eqs. [4] and [10] yield

$$
\begin{gathered}
\nabla^{* 2} \phi_{2}^{*}-\frac{(\kappa a)^{2}}{(1+\alpha)}\left[\exp \left(-\phi_{r} \phi_{1}^{*}\right)+\alpha \exp \left(\alpha \phi_{r} \phi_{1}^{*}\right)\right] \phi_{2}^{*} \\
=\frac{(\kappa a)^{2}}{(1+\alpha)}\left[\exp \left(-\phi_{r} \phi_{1}^{*}\right) g_{1}^{*}+\exp \left(\alpha \phi_{r} \phi_{1}^{*}\right) \alpha g_{2}^{*}\right] .
\end{gathered}
$$

The applied electric field is in the $Z$ direction, therefore, we have, for the scaled perturbed potential $\phi_{2}^{*}$,

$$
\begin{gathered}
\phi_{2}^{*}=-\mathbf{E}_{z}^{*} \cdot \mathbf{r}^{*}, \quad \eta=0 \text { and } \xi=0, \quad \text { or } \eta=0 \\
\frac{\partial \phi_{2}^{*}}{\partial \eta}=0, \quad \eta=\eta_{0} .
\end{gathered}
$$

The symmetric nature of the problem suggests that

$$
\frac{\partial \phi_{2}^{*}}{\partial \xi}=0, \quad \xi=0 \quad \text { or } \quad \xi=\pi
$$

In these expressions, $\mathbf{E}_{z}^{*}$ and $\mathbf{r}=r^{*} \cdot \mathbf{i}_{r}$ are respectively the scaled electric field and the direction vector where

$$
r^{*}=c^{*}\left(\frac{\cosh \eta+\cos \xi}{x}\right)^{1 / 2} .
$$

Substituting Eq. [8] into Eq. [3] gives

$$
\begin{aligned}
\nabla^{* 2} g_{1}^{*}-\phi_{r} \nabla^{*} \phi_{1}^{*} \cdot \nabla^{*} g_{1}^{*} & =\phi_{r}^{2} P e_{1} \mathbf{v}^{*} \cdot \nabla^{*} \phi_{1}^{*} \\
\nabla^{* 2} g_{2}^{*}+\alpha \phi_{r} \nabla^{*} \phi_{1}^{*} \cdot \nabla^{*} g_{2}^{*} & =\phi_{r}^{2} P e_{2} \mathbf{v}^{*} \cdot \nabla^{*} \phi_{1}^{*} .
\end{aligned}
$$

In these expressions $P e_{j}=\varepsilon\left(Z_{1} e / k_{B} T\right)^{2} / \eta D_{j}, j=1,2$, is the electric Peclet number of ionic species $j$, and $\mathbf{v}^{*}=\mathbf{v} / U_{E}$, $U_{E}=\left(\varepsilon \zeta_{k}^{2} / \eta a\right)$ is the magnitude of the velocity of an isolated particle based on Smoluchowski's theory when an electric field of strength $\left(\zeta_{a} / a\right)$ is applied,

Suppose that the perturbed electrical potential arises from the induced electric field vanishes at a point far away from particle and plane surface. Then

$$
g_{j}^{*}=-\phi_{2}^{*}, \quad \eta=0 \quad \text { and } \quad \xi=0, \quad \text { or } \quad \eta=0 .
$$

The surface of particle is impenetrable to ionic species, which implies that

$$
\frac{\partial g_{j}^{*}}{\partial \eta}=0, \quad \eta=\eta_{0}
$$

The symmetric nature of the problem requires that

$$
\frac{\partial g_{j}^{*}}{\partial \xi}=0, \quad \xi=0 \quad \text { or } \quad \xi=\pi
$$

In terms of stream function, Eq. [5] becomes, after taking curl,

$$
\mu \frac{\mathbf{i}_{\varphi}}{\omega} E^{4} \psi-\nabla \times(\rho \nabla \phi)=0
$$

where $\omega=c \sin \xi / x$ is the distance from the plane $\xi=\pi$. If we let $\mathbf{v}_{\eta}$ and $\mathbf{v}_{\xi}$ be the $\eta$ and $\xi$ components of velocity $\mathbf{v}$, then

$$
\begin{aligned}
& \mathbf{v}_{\eta}=\frac{x^{2}}{c^{2} \sin \xi} \frac{\partial \psi}{\partial \xi} \\
& \mathbf{v}_{\xi}=\frac{-x^{2}}{c^{2} \sin \xi} \frac{\partial \psi}{\partial \eta}
\end{aligned}
$$

Substituting Eq. [9] into Eq. [26] and rewriting the resultant expression in scaled form, we obtain

$$
E^{4} \psi^{*}=-(\kappa a)^{2} \frac{x \sin \xi}{c^{*}}\left(\frac{\partial \phi_{1}^{*}}{\partial \xi} \frac{\partial \phi_{2}^{*}}{\partial \eta}-\frac{\partial \phi_{1}^{*}}{\partial \eta} \frac{\partial \phi_{2}^{*}}{\partial \xi}\right) .
$$

Here, $\psi^{*}=\psi / U_{E} a$ is the scaled stream function, and $E^{4}=$ $E^{2} E^{2}$, with $E^{2}$ defined by

$$
E^{2}=\frac{x^{2}}{c^{* 2}}\left[\frac{\partial^{2}}{\partial \eta^{2}}+\frac{\partial^{2}}{\partial \xi^{2}}+\frac{\sinh \eta}{x} \frac{\partial}{\partial \eta}+\frac{1-\cos \xi \cosh \eta}{x \sin \xi} \frac{\partial}{\partial \xi}\right] .
$$

The following boundary conditions are assumed for the flow field:

$$
\begin{gathered}
\psi^{*}=\frac{-1}{2} \omega^{* 2} U^{*} \text { and } \frac{\partial \psi^{*}}{\partial \eta}=\frac{c^{* 2}}{x^{3}} \sin \xi \sinh \eta U^{*}, \quad \eta=\eta_{0} \\
\psi^{*}=0 \quad \text { and } \frac{\partial \psi^{*}}{\partial \xi}=0, \quad \xi=0 \quad \text { or } \quad \xi=\pi \\
\psi^{*}=0 \quad \text { and } \frac{\partial \psi^{*}}{\partial \eta}=0, \quad \eta=0 \\
\frac{\psi^{*}}{r^{* 2}} \rightarrow 0, \quad \eta=0 \quad \text { and } \xi=0
\end{gathered}
$$

In these expressions, $\omega^{*}=c^{*} \sin \xi / x$ and $U^{*}=U / U_{E}$. Equations [31]-[34] imply that the velocity of the particle is $\mathbf{U}$, and the fluid at infinity is stationary.

The forces acting on the particle include the electric force $F_{E z}$ and the hydrodynamic force $F_{D z}$, which can be evaluated respectively by

$$
F_{E z}=2 \pi \int_{0}^{\pi} \sigma(-\nabla \phi) \delta s
$$




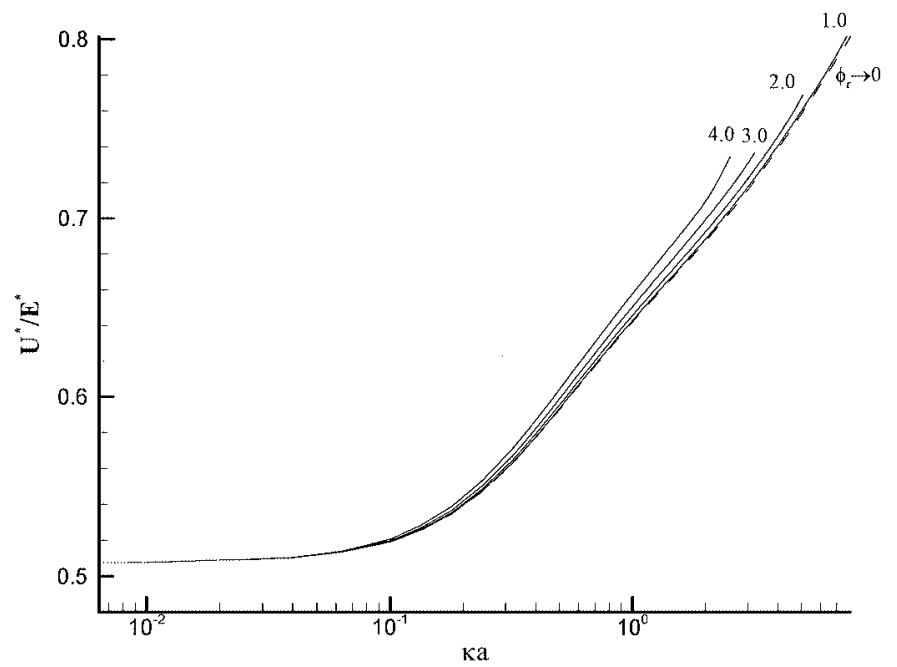

FIG. 2. Variation of scaled mobility $\left(U^{*} / E^{*}\right)$ as a function of $\kappa a$ at various scaled surface potential $\phi_{r}$ without considering DLP for the case $\alpha=1.0$ and $\eta_{0}=2.0$. The dashed line represents the result based on the linearized Eq. [4].

and

$$
F_{D z}=\mu \pi \int_{0}^{\pi} \omega^{3} \frac{\partial}{\partial n} \frac{E^{2} \psi}{\omega^{2}} \delta s-\pi \int_{0}^{\pi} \omega^{2} \rho_{e} \frac{\partial \phi}{\partial s} \delta s
$$

In these expressions $s$ represents the tangential surface, and the charge density on particle surface $\sigma$ can be evaluated by

$$
\sigma=\varepsilon \frac{x}{c} \frac{\partial \phi}{\partial \eta}
$$

Substituting Eq. [37] into Eq. [35] yields

$$
\begin{aligned}
F_{E z}^{*}= & 2 \pi \int_{0}^{\pi}-\frac{\sin \xi}{x} \frac{\partial \phi^{*}}{\partial \eta} \\
& \times\left((1-\cosh \eta \cos \xi) \frac{\partial \phi^{*}}{\partial \eta}-\sinh \eta \sin \xi \frac{\partial \phi^{*}}{\partial \xi}\right) d \xi
\end{aligned}
$$

Substituting Eq. [7a] into Eq. [41] gives

$$
\begin{aligned}
F_{D z}^{*}= & \pi \int_{0}^{\pi}-\omega^{* 3} \frac{\partial}{\partial \eta} \frac{E^{2} \psi^{*}}{\omega^{* 2}} d \xi-\frac{(\kappa a)^{2}}{(1+\alpha) \phi_{r}} \pi \\
& \times \int_{0}^{\pi} \omega^{* 2}\left[\exp \left(-\phi_{r} \phi_{1}^{*}\right)\left(1-\phi_{r}\left(\phi_{2}^{*}+g_{1}^{*}\right)\right)\right. \\
& +\exp \left(\alpha \phi_{r} \phi_{1}^{*}\left(1+\alpha \phi_{r}\left(\phi_{2}^{*}+g_{2}^{*}\right)\right) \frac{\partial \phi^{*}}{\partial \xi} d \xi .\right.
\end{aligned}
$$

The problem under consideration can be decomposed into two subproblems (6). In the first, the particle moves at constant velocity in the absence of the applied electric field, and in the second it is held constant under the external electric field. Let $f_{1}$ and $f_{2}$ be respectively the net forces acting on the particle in the first and second problems. We have $f_{1}=\chi U^{*}$ and $f_{2}=\beta E^{*}$. At steady state the net force acting on the particle vanishes, and, therefore, its mobility can be expressed as $U^{*} / E^{*}=-\beta / \chi$.

\section{RESULTS AND DISCUSSION}

The behavior of the system under consideration is examined through numerical simulation. A pseudo-spectral method based on Chebyshev polynomial (26), which is readily applicable to the present problem, is adopted for the resolution of the governing equations subject to the boundary conditions assumed.
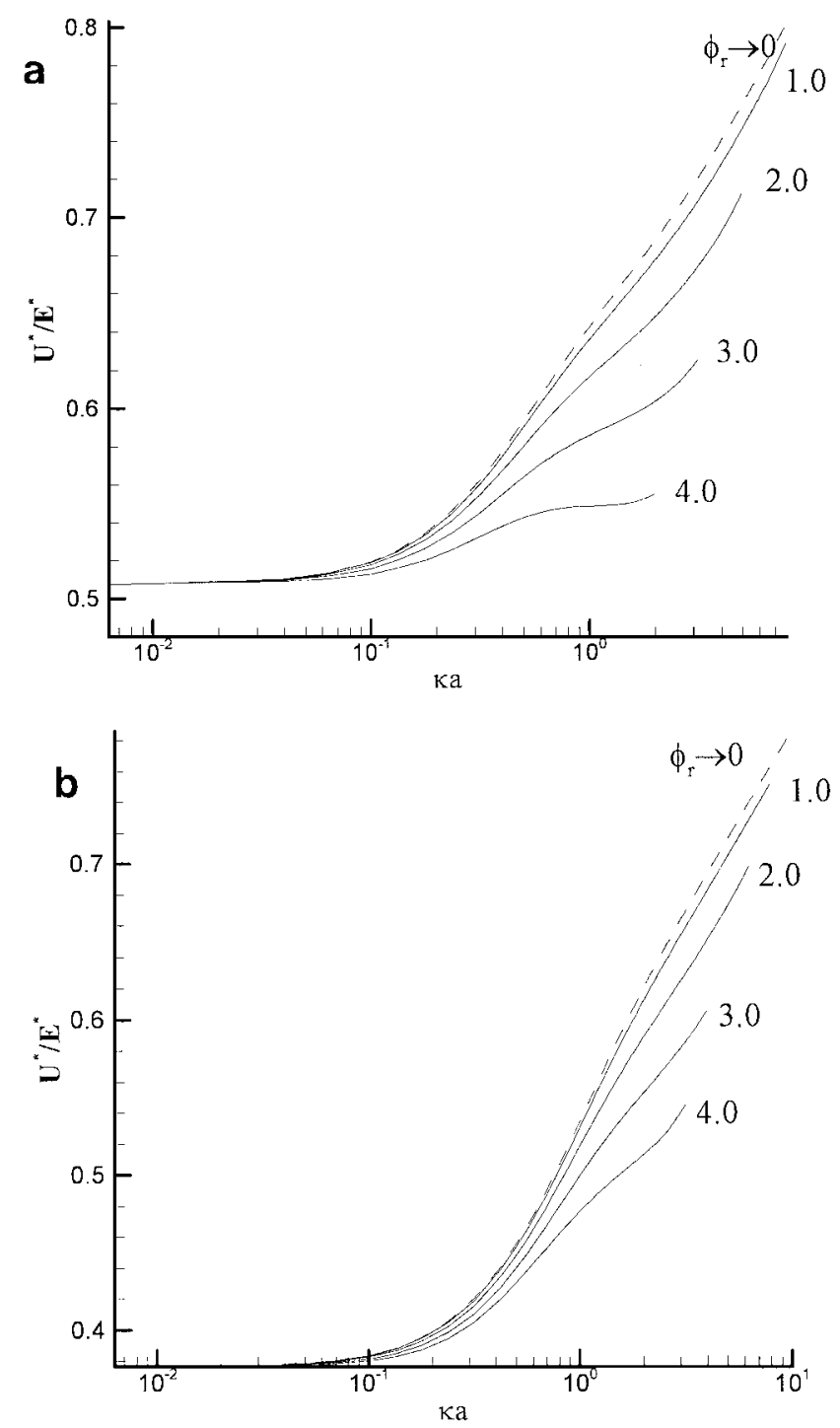

FIG. 3. Variation of scaled mobility $\left(U^{*} / E^{*}\right)$ as function of $\kappa a$ at various scaled surface potential $\phi_{r}$ for the case when DLP is considered with $P e_{1}=0.01$, $P e_{2}=0.01$, and $\alpha=1.0$ : (a) $\eta_{0}=2.0$; (b) $\eta_{0}=1.5$. 
The numerical procedure is introduced briefly in the Appendix. The method adopted has a fast rate of convergence, and the convergent properties are independent of the associated boundary conditions. Also, the mini-max property typically associated with the Chebyshev polynomial is maintained.

Figure 2 shows the variations of the scaled mobility $\left(U^{*} / E^{*}\right)$ as a function of $\kappa a$ at various scaled surface potential $\phi_{r}$ without considering DLP, which can be obtained by letting $g_{j}=0$ in Eq. [7], and the corresponding result when DLP is taken into account is illustrated in Fig. 3. For comparison, the results based on linearized Eq. [4], that is, those at low electrical potentials, are also presented in these figures. Figure 2 reveals that if $\kappa a$ is small (i.e., thick double layer), $\left(U^{*} / E^{*}\right)$ becomes independent of the surface potential of particle. This is because if the double layer is sufficiently thick, it will reach the planar surface. In this case, the movement of the particle is dominated by hydrodynamic conditions, and the effect of the electric field is less significant. As $\kappa a$ increases, the presence of the planar surface on the movement of the particle becomes less significant, and $\left(U^{*} / E^{*}\right)$ increases accordingly. Figure 2 also suggests that $\left(U^{*} / E^{*}\right)$ increases with the increase in the surface potential of particle, but the degree of increase is inappreciable. As can be seen in Fig. 3, the behavior of $\left(U^{*} / E^{*}\right)$ at small $\kappa a$ when the effect of DLP is taken into account is similar to that when it is neglected. The situation is different, however, if $\kappa a$ becomes large. This figure reveals that $\left(U^{*} / E^{*}\right)$ decreases with the increase in the surface potential of particle, and the degree of increase is appreciable. This is because the polarization of the double layer has the effect of inducing an internal electric field, which is in the inverse direction as that of the applied electric field. Since the strength of the induced electric field increases with the surface potential of the particle, $\left(U^{*} / E^{*}\right)$ decreases with the increase in $\phi_{r}$. This phenomenon can also be explained by the variation of $\beta$ as a function of $\kappa a$, as illustrated in Fig. 4. According to its

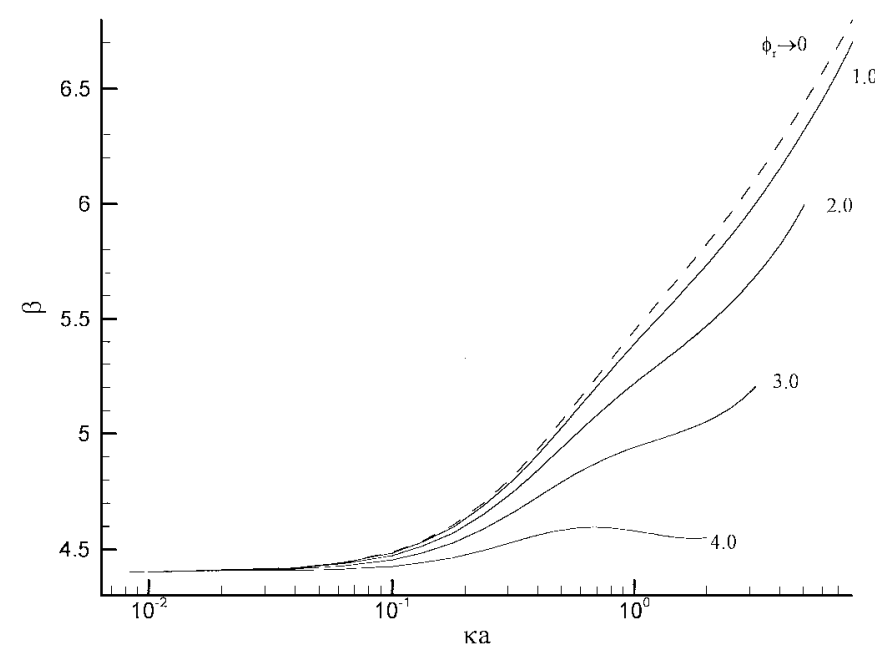

FIG. 4. Variation of $\beta$ as function of $\kappa a$ at various scaled surface potential $\phi_{r}$ for the case when DLP is considered with $P e_{1}=0.01, P e_{2}=0.01, \alpha=1.0$, and $\eta_{0}=2.0$.

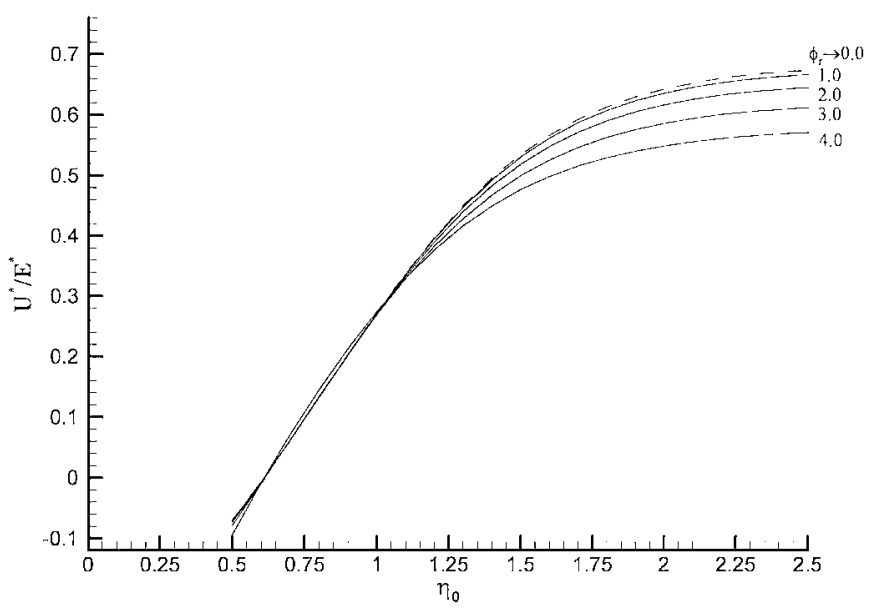

FIG. 5. Variation of scaled mobility $\left(U^{*} / E^{*}\right)$ as function of $\eta_{0}$ at various scaled surface potential $\phi_{r}$ for the case when DLP is considered with $P e_{1}=0.01$, $P e_{2}=0.01, \alpha=1.0$, and $\kappa a=1.0$.

definition, $\beta$ is a measure for the net force acting on a particle in problem 2. Figure 4 shows that if $\phi_{r}$ is fixed, $\beta$ increases with the increase in $\kappa a$. This is because the thinner the double layer, the smaller the resistant force experienced by a particle. Again, the presence of DLP has the effect of reducing the electric force arising from the applied electric field, and the larger the $\phi_{r}$ the more significant the effect DLP.

Figure 5 illustrates the variation of the scaled mobility $\left(U^{*} / E^{*}\right)$ as a function of $\eta_{0}$ at various scaled surface potential $\phi_{r}$ for the case when DLP is considered. According to its definition $\left(h / a=\cosh \eta_{0}\right), \eta_{0}$ is a measure for the distance between particle and surface, the smaller the $\eta_{0}$ the closer the distance. As can be seen from Fig. 5, $\left(U^{*} / E^{*}\right)$ decreases with the decrease in $\eta_{0}$. This is expected since the closer the distance between the particle and the surface the more significant the hydrodynamic hindrance of the movement of the former by the latter. Figure 5 suggests that if $\eta_{0}<1.0$, the mobility of a particle becomes insensitive to its surface potential. This is because if the distance between the particle and the surface is sufficiently close, the hydrodynamic effect dominates the electrical effect.

\section{CONCLUSION}

We conclude that under the condition in which a particle and a planar surface are not close, the surface potential of the farmer on its electrophoretic mobility is negligible when double layer polarization is neglected, but it becomes crucial if double layer polarization is taken into account. However, if the distance between the particle and the planar surface is sufficiently close, the hydrodynamic effect dominates, and the electric effects are insignificant.

\section{APPENDIX}

In the present case, the computational domain is two dimensional, and the pseudo-spectral method is applied in both the 
$\eta$ and $\xi$ directions. For instance, the $N$ th-order $\times M$ th-order approximation to the function $f_{N M}(\eta, \xi)$ is expressed by

$$
f_{N M}(\eta, \xi)=\sum_{i=0}^{N} \sum_{j=0}^{M} f_{N M}\left(\eta_{i}, \xi_{j}\right) h_{i}(\eta) h_{j}(\xi),
$$

where $f_{N M}\left(\eta_{i}, \xi_{j}\right)$ is the value of $f_{N M}$ at the $k$ th collocation point, where $k=[(N-1) i+j]$. The interpolation polynomials $h_{i}(\eta)$ and $h_{j}(\xi)$ depend on the collocation points and these points are determined by mapping the computational domain onto the square $[-1,1] \times[-1,1]$ through

$$
\begin{aligned}
& \eta=\frac{\eta_{0}}{2} y+\frac{\eta_{0}}{2} \\
& \xi=\frac{\pi}{2}(x+1) .
\end{aligned}
$$

The $N+1$ interpolation points in the interval $[-1,1]$ are chosen to be the extreme values of an $N$ th-order Chebyshev polynomial $T_{N}(y)$

$$
y_{j}=\cos \left(\frac{\pi j}{N}\right), \quad j=0,1, \ldots, N
$$

The corresponding interpolation polynomial $h_{i}(y)$ is

$$
h_{j}(y)=\frac{(-1)^{j+1}\left(1-y^{2}\right)\left(d T_{N}(y) / d y\right)}{c_{j} N^{2}\left(y-y_{j}\right)} \quad j=0,1, \ldots, N,
$$

where $c_{j}$ is defined by

$$
c_{j}= \begin{cases}2, & j=0, N \\ 1, & 1 \leq j \leq N-1 .\end{cases}
$$

Both the partial derivatives and the integration of $f_{N M}(\eta, \xi)$ are evaluated based on Eq. [A-1]. The corresponding nonlinear system is then solved with a Newton-Raphson iteration scheme.
Double precision is used throughout the computation, and grid independence is checked to ensure that the mesh used is fine enough. For each $\kappa a$ the calculations consist of $41 \times 41$ nodal points for $\left(N_{\eta} \times M_{\xi}\right)$.

\section{ACKNOWLEDGMENT}

This work is supported by the National Science Council of the Republic of China.

\section{REFERENCES}

1. Zydney, A. L., J. Colloid Interface Sci. 169, 476 (1995).

2. Lee, E., Chu, J. W., and Hsu, J. P., J. Colloid Interface Sci. 196, 316 (1997).

3. Lee, E., Chu, J. W., and Hsu, J. P., J. Colloid Interface Sci. 205, 65 (1998).

4. Morrison, F. A., and Stukel, J. J., J. Colloid Interface Sci. 33, 88 (1969).

5. Keh, H. J., and Lien, L. C., J. Chin. Inst. Chem. Eng. 20, 283 (1989).

6. Keh, H. J., and Anderson, J. L., J. Fluid Mech. 153, 417 (1985).

7. Ennis, J., and Anderson, J. L., J. Colloid Interface Sci. 185, 497 (1997).

8. Feng, J. J., and Wu, W. Y., J. Fluid Mech. 264, 41 (1994).

9. Shugai, A. A., and Carnie, S. L., J. Colloid Interface Sci. 213, 298 (1999).

10. Tang, Y. P., Chih, M. H., Lee, E., and Hsu, J. P., J. Colloid Interface Sci. 242, 121 (2001).

11. Von Smoluchowski, M., Z. Phys. Chem. 92, 129 (1918).

12. Huckel, E., Phys. Z. 25, 204 (1924).

13. Henry, D. C., Proc. R. Soc. London Ser. A 133, 106 (1931).

14. Booth, F., Proc. R. Soc. London Ser. A 203, 514 (1950).

15. Wiersema, P. H., Loeb, A. L., and Overbeek, J. Th. G., J. Colloid Interface Sci. 22, 78 (1966).

16. O'Brien, R. W., and White, L. R., J. Chem. Soc. Faraday Trans. 2 74, 1607 (1978).

17. O'Brien, R. W., and Hunter, R. J., Can. J. Chem. 59, 1878 (1981).

18. Ohshima, H., Healy, T. W., and White, L. R., J. Chem. Soc. Faraday Trans. 2 79, 1613 (1983).

19. Ohshima, H., Adv. Colloid Interface Sci. 62, 189 (1995).

20. Levine, S., and Neale, G. H., J. Colloid Interface Sci. 47, 520 (1974).

21. Kozak, M. W., and Davis, E. J., J. Colloid Interface Sci. 127, 497 (1989).

22. Kozak, M. W., and Davis, E. J., J. Colloid Interface Sci. 129, 166 (1989).

23. Ohshima, H., J. Colloid Interface Sci. 188, 481 (1997).

24. Lee, E., Chu, J. W., and Hsu, J. P., J. Colloid Interface Sci. 209, 240 (1999).

25. Happel, J., "Low Reynolds Number Hydrodynamics," Noordhoff, Leyden, The Netherlands, 1973.

26. Canuto, C., Hussaini, M. Y., Quarteroni, A., and Zang, T. A., "Spectral Methods in Fluid Dynamics," Springer-Verlag, Berlin/ New York, 1986. 